\title{
Prehospital Hemorrhage Assessment Criteria: A Concise Review
}

\author{
Sara Di Carlo, MD, PhD — Giuseppe Cavallaro, MD, PhD — Kenia Palomeque, MS $\square$ Maurizio Cardi, MD, PhD \\ Giuseppe Sica, MD, PhD — Piero Rossi, MD, PhD $\square$ Simone Sibio, MD, PhD
}

\begin{abstract}
Objective: Early assessment of the clinical status of trauma patients is crucial for guiding the treatment strategy, and it requires a rapid and systematic approach. The aim of this report is to critically review the assessment parameters currently used in the prehospital setting to quantify blood loss in trauma.

Data Sources: Studies regarding hemorrhagic shock in trauma were pooled from PubMed, EMBASE, and Cochrane databases using key words such as "hemorrhagic shock," "vital signs evaluation," "trauma," "blood loss," and "emergency medical service," alone or combined.

Study Selection: Articles published since 2009 in English and Italian were considered eligible if containing data on assessment parameters in blood loss in adults.
\end{abstract}

orldwide, trauma is the leading cause of death and disability in patients younger than 40 years, and it accounts for approximately $8 \%$ of all deaths, causing an enormous socioeconomic impact with regard to the costs of treatment and rehabilitation (World Health Organization, 2021). Hemorrhage is among the main causes of death in trauma, which can be exacerbated by inaccurate recognition or delayed treatment (Dunham, Sartorius, Laing, Bruce, \& Clarke, 2017; Lui et al., 2018). Hemorrhagic shock pathophysiology and compensatory mechanisms contribute to the masking of signs of clinical deterioration. Thus, knowledge of underlying pathophysiology assists the clinician's interpretation of assessment parameters.

\section{PATHOPHYSIOLOGY OF HEMORRHAGE}

Massive brisk intravascular volume loss leads to hemodynamic instability, decreased oxygen delivery, reduced

Author Affiliations: Department of Surgery, Tor Vergata University of Rome, Viale Oxford, Rome, Italy (Drs Di Carlo, Sica, and Rossi); and Department of Surgery "Pietro Valdoni," University "Sapienza" of Rome, Viale del Policlinico, Rome, Italy (Drs Cavallaro, Cardi, and Sibio and Ms Palomeque).

Data and materials are available upon request.

The authors declare no conflicts of interest.

Correspondence: Simone Sibio, MD, PhD, Department of Surgery, "Viale del Policlinico", University "Sapienza" of Rome, "Pietro Valdoni," Via Lancisi 2_00161 Rome, Italy (simone.sibio@uniroma1.it).

DOI: 10.1097/JTN.0000000000000608
Data Extraction: Sixteen articles matching the inclusion criteria were considered in our study.

Data Synthesis: Current prehospital assessment measures lack precise correlation with blood loss.

Conclusions: Traditional assessment parameters such as heart rate, systolic blood pressure, shock index, and Glasgow Coma Scale score often lag in providing accurate blood loss assessment. The current literature supports the need for a noninvasive, continuously monitored assessment parameter to identify early shock in the prehospital setting.

\section{Key Words}

Blood loss, Emergency, Exsanguination, Hemorrhagic shock, Prehospital care, Quantitative measures, Trauma

tissue perfusion, cellular hypoxia, organ damage, and death. A significant loss in circulating blood volume can depress cardiac output hence reducing organ perfusion pressure. A decrease in arterial pressure stimulates sympathetic nerve activity. Catecholamines, antidiuretic hormone, and atrial natriuretic receptors induce vasoconstriction of arterioles and muscular arteries, increasing vascular resistance leading to a compensatory elevation in arterial pressure. With the depletion of intravascular volume and the reduced oxygen availability in the tissues, mitochondria are no longer able to sustain aerobic metabolism for energy production and switch to anaerobic metabolism. In aerobic conditions, systemic oxygen utilization is proportional to the metabolic rate, and it varies according to the body's needs. During the shock, prostaglandins, adenosine, and nitric oxide tend to lower vascular resistance leading to hypoxic redistribution of blood flow to all organs. Under these conditions, a failure of the protonic pumps and the alteration of ion transport lead to a loss of the membrane integrity, depletion of cellular energy, acidosis, and loss of adenine nucleotides from the cell. This mechanism should maintain the perfusion pressure by increasing cardiac output. As a result of hypovolemia and tissue hypoxia, the ventilation increases (tachypnea) to compensate for the metabolic acidosis caused by carbon dioxide production. The decrease of blood flow to the renal and splanchnic vasculature contributes to a further drop of the systolic blood pressure (SBP) (Cannon, 2018; Convertino, Wirt, Glenn, \& Lein, 2016). 


\begin{tabular}{|c|c|c|c|c|}
\hline Shock Class Group & 1 & 2 & 3 & 4 \\
\hline HR (BPM) & $<100$ & 100-119 & 120-139 & $>140$ \\
\hline $\mathrm{SBP}(\mathrm{mm} \mathrm{Hg})$ & $>110$ & 100-109 & 90-99 & $<90$ \\
\hline GCS & 15 & 15 & $12-14$ & $<12$ \\
\hline
\end{tabular}

Prehospital assessment of hemorrhage is important because it is potentially the most critical time to detect and intervene with damage control measures. The first approach to the trauma patient occurs with assessment of the mechanism of injury to identify potential patterns of injury (Cardi et al., 2019; Frank et al., 2010). Time is crucial in trauma. Prehospital care is prioritized with lifesaving scene management and prompt transport to the most appropriate facility, often characterized as occurring within the "golden hour" (Wyen et al., 2013).

Blood loss estimation is notoriously challenging and consistently reported as unreliable (Ashburn, Harrison, Ham, \& Strote, 2012). Hemorrhage classification systems help clinicians rapidly and systematically focus assessment of the bleeding patient. The most commonly taught hemorrhagic shock classification is from Advanced Trauma Life Support (ATLS); see Table 1 (American College of Surgeons Committee on Trauma, 2021). However, evidence challenges the accuracy of the ATLS shock classification (Guly et al., 2011; Mutschler, Nienaber, Brockamp, et al., 2013; Mutschler, Nienaber, et al., 2014; Mutschler, Paffrath, et al., 2014; Reisner et al., 2018). The ATLS classification is based on average or standardized responses of static variables maintained by the body's mechanisms to compensate for blood loss. However, not all patients compensate equally. What is needed is an individualized and continuous assessment of the patient's progression toward shock that considers the patient's total degree of compensation to identify those patients deteriorating into early shock. Prehospital estimation of blood loss is crucial, yet tools to predict blood loss and need for transfusion are often time-consuming and impractical in the prehospital setting (Olaussen, Blackburn, Mitra, \& Fitzgerald, 2014; Van Sickle et al., 2013). Prompt shock recognition is essential to apply damage control resuscitation to reverse the conditions that exacerbate hemorrhage (Tonglet, Minon, Seidel, Poplavsky, \& Vergnion, 2014).

\section{OBJECTIVE}

The aim of this study is to critically review the assessment parameters currently used in the prehospital setting to quantify blood loss in trauma.

\section{METHODS}

A systematic search was performed of the PubMed, EMBASE, and Cochrane databases using the key words "hemorrhagic shock," "vital signs scale evaluation," "trauma," "blood loss," and "emergency medical service," alone or embedded. All articles published since 2009 matching with the search terms were analyzed. Only those studies concerning the adult trauma population and written in English or Italian were considered. References of the included studies were analyzed to find additional relevant articles. Articles were considered eligible if containing data on assessment parameters and blood loss in trauma patients undergoing hypovolemic shock. Titles and abstracts underwent a double-blinded evaluation to select articles for full-text reading. A senior researcher acted as supervisor in case of discordance. A final selection of articles was provided and underwent full text reading.

\section{RESULTS}

A total of 16 articles were included in the study, which identified five assessment measures used for prehospital estimation of blood loss. The measures include heart rate (HR), SBP, Shock Index (SI), Glasgow Coma Scale (GCS) score, and visual estimation of blood loss (see Table 2).

\section{Patient Characteristics}

Other factors can also influence the accuracy of data provided by assessment measures. Among them, the most important in altering clinical values are those depending on patient characteristics and treatment, such as the following:

- Age: Patients older than 55 years with normal vital signs cannot rule out impending shock. An HR greater than 100 and SBP of 100-110 may represent hypoperfusion (Heffernan et al., 2010), and the SI can increase from 0.79 to 0.81 (Zarzaur, Croce, Fischer, Magnotti, \& Fabian, 2008)

- Comorbidities: Neurological, cardiovascular, autoimmune

- Medications: $\beta$-blockers or neurological drugs can underestimate the shock 


\begin{tabular}{|c|c|c|c|}
\hline Authors & Scale & Parameters & Author Opinion \\
\hline Reisner et al. (2018) & ATLS & $\mathrm{HR}, \mathrm{SBP}, \mathrm{BL}$ & Changes appear to be subtle \\
\hline \multicolumn{4}{|l|}{ Guly et al. (2011) } \\
\hline Mutschler et al. (2013) & $\mathrm{SI}(\mathrm{HR} / \mathrm{SBP})$ & 4 classes: & $\mathrm{SI} \geq 1$ good parameter to predict $\uparrow$ mortality and $\uparrow$ bt \\
\hline Pacagnella et al. (2013) & & I: $\leq 0.6$ & \\
\hline Van Sickle et al. (2013) & & II: $\geq 0.6$ to $\leq 1$ & \\
\hline Pandit et al. (2014) & & III: $\geq 1$ to $\leq 1.4$ & \\
\hline Olaussen et al. (2014) & & IV: $\geq 1.4$ & \\
\hline Merlin et al. (2009) & MAR method & Clenched fist $=20-\mathrm{ml}$ blood & Improves accuracy and precision \\
\hline
\end{tabular}

- Lifestyle: Fit and active patients with physiological bradycardia achieve HR compensation values of up to 80-90 bpm

- Pregnancy: May lead to an underestimation of the bleeding due to the physiological changes

- Fluids: Prehospital administration of $1 \mathrm{~L}$ of fluids shifts the SI cutoff values to 1.0 and greater $(1.1[S D=$ $0.6]$ vs. $0.7[S D=0.2], p>.001$; Mitra, Fitzgerald, \& Chan, 2013).

\section{Assessment Parameters}

\section{$H R$ and SBP}

The inverse relationship of HR to SBP is commonly assumed to occur in hemorrhage and commonly taught to providers to assess, yet it does not consistently occur among bleeding patients (Pacagnella et al., 2013). Using standardized vital sign norms does not distinguish those patients who can compensate well versus those who cannot, leading to delayed identification of shock progression. Mounting evidence challenge the dogma that vital signs changes can identify early blood loss.

Mizushima, Ueno, Watanabe, Ishikawa, and Matsuoka (2011) retrospectively analyzed a large database of trauma patients with base deficit and lactate levels indicating hemorrhage; a normal HR was a predictor of poor prognosis. Heart rate is nonspecific to blood loss and influenced by other factors such as age, pain, temperature, and medications, and thus cannot be considered a reliable parameter. Indeed, bradycardia or normal HR is reported to be more common in hemorrhage than expected. Ley, Salim, Kohanzadeh, Mirocha, and Margulies (2009) found the incidence of relative bradycardia in $44 \%$ of all hypotensive trauma patients on a large data set of more than 130,000 patients. They also found that bradycardia is an indicator of higher mortality.
Similar results were reported by Riordan, Norris, Jenkins, and Morris (2009) in a study of 2,178 trauma patients admitted to the intensive care unit, in which reduced HR was associated with worsening prognosis and higher mortality, especially in patients with isolated head injury or penetrating trauma. Guly et al. (2011), in a study of blunt and penetrating trauma, demonstrated an average HR and blood pressure (in patients with blood loss of $>40 \%$ of the volume) lower than the values reported in the ATLS classification for the same hypovolemia (Class 4).

Moreover, a varied correlation between vital signs and actual blood loss was highlighted by Reisner et al. (2018). Their retrospective comparative analysis studied HR changes in adult trauma with hemorrhage during either prehospital or emergency department care. Hemorrhage patients were divided into two groups, those with a normal HR and those with tachycardia. They demonstrated that some trauma patients with hemorrhage are continuously tachycardic whereas others have a normal HR. For both cohorts, hypotension developed within 30 min without any consistent increase in HR. Tachycardia was not specific for hemorrhage.

\section{Shock Index}

Shock index, defined as the HR divided by SBP with values of 1 or more considered abnormal, is another parameter used to assess blood loss. Shock index is gaining favor as a predictor for transfusion or prognostic factor in major trauma (Mutschler, Nienaber, Brockamp, et al., 2013; Pandit et al., 2014). Olaussen et al. (2014) demonstrated the SI's effectiveness in identifying severe bleeding in the prehospital setting. However, this observation was not confirmed by Van Sickle et al. (2013), who found that SI indicated hypovolemia only in the advanced stages of reduced central blood volume; moreover, they 
established that this index was not able to estimate the blood volume loss or to establish the patient's residual compensation capacity. The main limit of this study was the small sample size of 15 patients.

\section{Glasgow Coma Scale}

According to the ATLS classification, GCS, together with SBP and HR, is considered one of the parameters evaluated in the prehospital setting and on admission to rule out hemorrhagic shock. The descriptors slightly anxious and mildly anxious were considered a GCS score of 15, whereas anxious or confused was considered a GCS score of 12-14 and confused or lethargic as GCS score of less than 12. Changes in the GCS are considered reliable indicators of hemorrhagic shock for some authors (Vishwanathan, Chhajwani, Gupta, \& Vaishya, 2020). Some reports found a correlation between hypotension and GCS reduction for patients with injury severity score of more than 15 (Guly et al., 2011), whereas other studies with larger data sets find a lack of a clear correlation between GCS and changes in SBP and HR as well as the onset and progression of hypotension (Mutschler, Nienaber, Munzberg, et al., 2013). Furthermore, prehospital GCS could be influenced by prehospital intubation, whose rates vary across providers.

\section{Visual Estimation of Blood Loss}

Analysis of large data sets suggests a correlation between the volume of red blood cell loss and the blood products needed to reestablish hemodynamic balance. High severity bleeding ( $>15 \%$ of circulating blood volume) in trauma leads to an average of 6-10 units of blood transfused per patient in $24 \mathrm{hr}$, especially for those who experience hypotension and tachycardia (Reisner et al., 2018; Weeber, Hunter, Van Hoving, \& Lategan, 2018). Even from these studies, inaccurate evaluation of the blood loss emerges; for values less than $300 \mathrm{ml}$, there was a tendency to overestimate the quantity, whereas for volumes between $400 \mathrm{ml}$ and $1,500 \mathrm{ml}$, there was an underestimation (Patton, Funk, Mcerlean, \& Bartfield, 2001). This study found that emergency medical service personnel were unable or strongly inaccurate in estimating blood loss.

Similar results were reported by Frank et al. (2010), who used a prospective blinded, observational design and asked the participants to visually estimate the amount of blood loss in six simulated trauma scenarios. Both paramedics and emergency physicians overestimated the amount of blood loss when the assessment parameters showed instability (low blood pressure, high HR). Small volumes were overestimated for both stable and unstable patients, whereas higher volumes tended to be underestimated.

The accuracy of blood loss evaluation was not influenced by profession (emergency physician or paramedic), gender, or experience level. Ashburn et al. (2012) demonstrated that such estimates could be misleading if used in clinical decision making. Williams and Boyle (2007) described the inaccuracy of blood loss estimation given by undergraduate paramedic students for both absorbent and impermeable surfaces. Finally, Phillips, Friberg, Lantz Cronqvist, Jonson, and Prytz (2020) investigated the ability of laypeople to assess blood loss and also found overestimation of small volumes (from 50 to $200 \mathrm{ml}$ ) and underestimation of large volumes (from 400 to $1,900 \mathrm{ml}$ ). Moreover, the underestimation was more common for female victims than for male victims.

One novel study sought to identify a comparable estimate of blood loss using the size of a clenched fist (Merlin, Alter, Raffel, \& Pryor, 2009). Called the MAR method, a clenched fist was found to approximate roughly $20 \mathrm{ml}$ of blood loss. Merlin et al. (2009) performed an unblinded crossover trial of 74 health care professionals' estimation of blood loss in two scenarios ( $75 \mathrm{ml}$ and $750 \mathrm{ml})$. A comparison before and after a 1-min education session on the MAR method demonstrated significantly improved blood loss estimation.

\section{DISCUSSION}

Humans compensate for blood loss through a cascade of physiologic responses, contributing to unrecognized hemorrhagic shock. Our review finds a lack of reliable assessment measures to identify early blood loss in the prehospital setting. Although advances in trauma technology, practice, and guidelines continuously update, the prehospital ability to assess hemorrhage has not changed for decades. Accurate estimation of blood loss in trauma is paramount, yet neither medical personnel nor paramedics are instructed how to estimate blood volume loss and have no specific measure to assess it. The cornerstone of hemorrhagic trauma management is the reestablishment of hemodynamic stability by controlling the source of bleeding and reintegrating the volume loss with balanced transfusions, all of which begin with prehospital recognition of blood loss.

\section{Future Directions}

Emerging research explores new technology that may help with prehospital hemorrhage recognition.

\section{Ultrasound}

The role of ultrasound-focused assessment with sonography in trauma is emerging as a tool for the prehospital environment and holds promise (Kalkwarf, Goodman, Press, Wade, \& Cotton, 2021; Pietersen et al., 2021). Its role is operator-dependent, and it has some limitations, such as difficulties in exploring some deep regions and poor detection of some organ injuries depending on the patients' habitus (Miele et al., 2016). Therefore, a negative examination does not rule out injuries and must be 
verified by a computed tomographic scan (Stengel et al., 2018). However, it has several advantages, such as portability, speed, and the lack of ionizing radiation exposure.

\section{End-tidal Carbon Dioxide}

End-tidal carbon dioxide $\left(\mathrm{ETCO}_{2}\right)$ is the measurement of alveolar carbon dioxide concentration at end-expiration. It is measured noninvasively either in the ventilator circuit of intubated patients or with specialized nasal cannulas in nonintubated patients. End-tidal carbon dioxide is widely available across emergency medical services agencies and holds promise as prehospital levels have been shown to correlate with mortality and the need for massive transfusion or early death (Campion et al., 2020).

\section{Compensatory Reserve Measurement}

There is ongoing military research to develop wearable sensors to measure a patient's compensatory reserve defined as a composite or sum total of the patient's individual compensatory responses (tachycardia, vasoconstriction, breathing) to hemorrhage using real-time measurement of arterial waveforms. The measurement of a patient's compensatory reserve is more sensitive and specific in monitoring physiological status than other measures, including HR variability and HR complexity, and hold promise as a future shock measure as technology advances (Convertino et al., 2016; Convertino et al., 2020. Schlotman et al., 2020).

\section{Limitations}

Our review has some limitations. First is the limited number of articles that address the correlation between assessment parameters and blood loss or the classification or quantification of blood loss in a standardized and precise manner. Only one of 16 articles provided a method for estimating blood loss. Most of the articles were retrospective; the data were often incomplete or did not take into account the hospital treatment; only six out of 16 articles examined the visual estimation of the blood losses by the health care staff (prehospital and hospital setting); the majority of these articles used a blood-like product, which lacked all its typical characteristics (coagulation, viscosity, etc.) to simulate the scenario; and finally, out of 16 articles, only seven used simulation scenarios.

\section{CONCLUSIONS}

Available parameters to assess early blood loss in the prehospital setting are insensitive and nonspecific. Blood loss assessment is restricted by use of legacy vital signs that remain unchanged during the compensatory stage of shock. The correlation between changes in vital signs and early hemorrhage is highly variable and dependent on interindividual responses. Using static standardized vital sign norms does little to identify the patient early in compensated shock. Future research aims to validate an easy, precise, and individualized continuous assessment tool for estimating blood loss in the prehospital setting. Research on wearable or catheter-based systems holds promise to assess blood volume status in the future (Convertino et al., 2020; Zia, Kimball, Rolfes, Hahn, \& Inan, 2020).

\section{KEY POINTS}

- Prehospital assessment measures for hemorrhage have largely remained unchanged for decades.

- Available parameters to assess early blood loss in the prehospital setting are insensitive and nonspecific.

- The literature supports the need for a noninvasive, continuously monitored assessment parameter that incorporates individualized patient compensatory changes to detect early hemorrhagic shock in the prehospital setting.

\section{REFERENCES}

American College of Surgeons Committee on Trauma. (2021). Advanced trauma life support ATLS student course manual (10th ed.). Chicago, IL: American College of Surgeons.

Ashburn, J. C., Harrison, T., Ham, J. J., \& Strote, J. (2012). Emergency physician estimation of blood loss. The Western Journal of Emergency Medicine, 13(4), 376-379. doi:10.5811/ westjem.2011.9.6669

Campion, E. M., Cralley, A., Robinson, C., Sauaia, A., Pieracci, F. M., Lawless, R. A., ... Burlew, C. C. (2020). Prehospital endtidal carbon dioxide predicts massive transfusion and death following trauma. The Journal of Trauma and Acute Care Surgery, 89(4), 703-707. doi:10.1097/TA.0000000000002846

Cannon, J. W. (2018). Hemorrhagic shock. The New England Journal of Medicine, 378(4), 370-379. doi:10.1056/NEJMra1705649

Cardi, M., Ibrahim, K., Alizai, S. W., Mohammad, H., Garatti, M., Rainone, A., ... Sibio, S. (2019). Injury patterns and causes of death in 953 patients with penetrating abdominal war wounds in a civilian independent non-governmental organization hospital in Lashkargah, Afghanistan. World Journal of Emergency Surgery, 14(51), 1-8. doi:10.1186/s13017-0190272-z. eCollection 2019

Convertino, V. A., Schauer, S. G., Weitzel, E. K., Cardin, S., Stackle, M. E., Talley, M. J., ... Inan, O. T. (2020). Wearable sensors incorporating compensatory reserve measurement for advancing physiological monitoring in critically injured trauma patients. Sensors (Basel), 20(22), 1-24. doi:10.3390/s20226413

Convertino, V. A., Wirt, M. D., Glenn, J. F., \& Lein, B. C. (2016). The Compensatory Reserve for early and accurate prediction of hemodynamic compromise: A review of the underlying physiology. Shock, 45(6), 580-590. doi:10.1097/ SHK.0000000000000559

Dunham, M. P., Sartorius, B., Laing, G. L., Bruce, J. L., \& Clarke, D. L. (2017). A comparison of base deficit and vital signs in the early assessment of patients with penetrating trauma in a high burden setting. Injury, 48(9), 1972-1977. doi:10.1016/j. injury.2017.06.011

Frank, M., Schmucker, U., Stengel, D., Fisher, L., Lange, J., Grossjohann, R., ... Matthes, G. (2010). Proper estimation of blood loss on scene of trauma: Tool or tale? The Journal of Trauma, 69(5), 1191-1195. doi:10.1097/TA.0b013e3181c452e7

Guly, H. R., Bouamra, O., Spiers, M., Dark, P., Coats, T., Lecky, F. E.; Trauma Audit and Research Network. (2011). Vital signs and estimated blood loss in patients with major trauma: 
Testing the validity of the ATLS classification of hypovolemic shock. Resuscitation, 82(5), 556-559. doi:10.1016/j. resuscitation.2011.01.013

Heffernan, D. S., Thakkar, R. K., Monaghan, S. F., Ravindran, R., Adams, C. A., Jr., Kozloff, M. S., ... Cioffi, W. G. (2010). Normal presenting vital signs are unreliable in geriatric blunt trauma victims. The Journal of Trauma, 69(4), 813-820. doi:10.1097/ TA.0b013e3181f41af8

Kalkwarf, K. J., Goodman, M. D., Press, G. M., Wade, C. E., \& Cotton, B. A. (2021). Prehospital ABC score accurately forecasts patients who will require immediate resource utilization. Southern Medical Journal, 114(4), 193-198. doi:10.14423/ SMJ.0000000000001236

Ley, E. J., Salim, A., Kohanzadeh, S., Mirocha, J., \& Margulies, D. R. (2009). Relative bradycardia in hypotensive trauma patients A reappraisal. The Journal of Trauma, 675), 1051-1054. doi:10.1097/TA.0b013e3181bba222

Lui, C. T., Wong, O. F., Tsui, K. L., Kam, C. W., Li, S. M., Cheng, M., \& Leung, K. K. G. (2018). Predictive model integrating dynamic parameters for massive blood transfusion in major trauma patients: The Dynamic MBT Score. American Journal of Emergency Medicine, 36(8), 1444-1450. doi:10.1016/j. ajem.2018.01.009

Merlin, M. A., Alter, S. M., Raffel, B., \& Pryor, P. W. (2009) External blood loss estimation using the MAR method. The American Journal of Emergency Medicine, 27(9), 1085-1089. doi:10.1016/j.ajem.2008.07.039

Miele, V., Piccolo, C. L., Galluzzo, M., Ianniello, S., Sessa, B., \& Trinci, M. (2016). Contrast enhanced-ultrasound (CEUS) in blunt abdominal trauma. The British Journal of Radiology, 89(1061), 20150823. doi:10.1259/bjr.20150823

Mitra, B., Fitzgerald, M., \& Chan, J. (2013). The utility of a shock index $\geq 1$ as an indication for prehospital oxygen carrier administration in major trauma. Injury, 45(1), 61-65. doi:10.1016/j.injury.2013.01.010

Mizushima, Y., Ueno, M., Watanabe, H., Ishikawa, K., \& Matsuoka, T. (2011). Discrepancy between heart rate and makers of hypoperfusion is a predictor of mortality in trauma patients. The Journal of Trauma, 71(4), 789-792. doi:10.1097/ TA.0b013e31822f 7 bbd

Mutschler, M., Nienaber, S. U., Brockamp, T., Wafaisade, A., Wyen, H., Peiniger, S., ... TraumaRegister DGU. (2013). A critical reappraisal of the ATLS classification of hypovolemic shock Does it really reflect clinical reality? Resuscitation, 84(3), 309313. doi:10.1016/i.resuscitation.2012.07.012

Mutschler, M., Nienaber, U., Munzberg, M., Fabian, T., Paffrath, T. Wolfl, C., ... Maegele, M. (2014). Assessment of hypovolemic shock at scene: Is the PHTLS classification of hypovolemic shock really valid? Emergency Medicine Journal, 31(1), 35-40. doi:10.1136/emermed-2012-202130

Mutschler, M., Nienaber, U., Munzberg, M., Wolfl, C., Schoechl, H., Paffrath, T., ... TraumaRegister DGU. (2013). The shock index revisited? A fast guide to transfusion requirement? A retrospective analysis on 21853 patients derived from the Trauma Register DGU. Critical Care (London, England), 17(4), R172. doi:10.1186/cc12851

Mutschler, M., Paffrath, T., Wolfl, C., Probst, C., Nienaber, U., Schipper, I. B., ... Maegele, M. (2014). The ATLS classification of hypovolemic shock: A well established teaching tool on the edge. Injury, 45(S3), 5-8. doi:10.1016/j.injury.2014.08.015

Olaussen, A., Blackburn, T., Mitra, B., \& Fitzgerald, M. (2014). Shock Index for prediction of critical bleeding post-trauma: A systematic review. Emergency Medicine Australasia, 26(3), 223-228. doi:10.1111/1742-6723.12232

Pacagnella, R. C., Souza, J. P., Durocher, J., Perel, P., Blum, J., Winikoff, B., \& Gulmezoglu, A. M. (2013). A systematic review of the relationship between blood loss and clinical signs. PLoS One, 8(3), e57594. doi:10.1371/journal.pone.0057594
Pandit, V., Rhee, P., Hashmi, A., Kulvatunyou, N., Tang, A., Khalil, M., ... Joseph, B. (2014). Shock index predicts mortality in geriatric trauma patients: An analysis of the National Trauma Data Bank. The Journal of Trauma and Acute Care Surgery, 76(4), 1111-1115. doi:10.1097/TA.0000000000000160

Patton, K., Funk, D. L., Mcerlean, M., \& Bartfield, J. M. (2001) Accuracy of estimation of external blood loss by EMS personnel. Journal of Trauma, 50(5), 914-916. doi:10.1097/00005373200105000-00023

Phillips, R., Friberg, M., Lantz Cronqvist, M., Jonson, C. O., \& Prytz, E. (2020). Visual estimates of blood loss by medical laypeople: Effects of blood loss volume, victim gender, and perspective. PLoS One, 15(11), e0242096. doi:10.1371/journal.pone. 0242096

Pietersen, P. I., Mikkelsen, S., Lassen, A. T., Helmerik, S., Jorgensen, G., Nadim, G., ... Laursen, C. B. (2021). Quality of focused thoracic ultrasound performed by emergency medical technicians and paramedics in a prehospital setting: A feasibility study. Scandinavian Journal of Trauma Resuscitation and Emergency Medicine, 29(1), 40. doi:10.1186/s13049-021-008568

Reisner, A. T., Edla, S., Liu, J., Liu, J., Khitrov, M. Y., \& Reifman, J (2018). Tachycardic and non-tachycardic responses in trauma patients with haemorrhagic injuries. Injury, 49(9), 1654-1660. doi:10.1016/j.injury.2018.04.032

Riordan, W. P., Jr., Norris, P. R., Jenkins, J. M., \& Morris, J. A. Jr (2009). Early loss of heart rate complexity predicts mortality regardless of mechanism, anatomic location, or severity of injury in 2178 trauma patients. Journal of Surgical Research, 156(2), 283-289. doi:10.1016/j.jss.2009.03.086

Schlotman, T. E., Suresh, M. R., Koons, N. J., Howard, J. T., Schiller, A. M., Cardin, S., \& Convertino, V. A. (2020). Predictors of hemodynamic decompensation in progressive hypovolemia: Compensatory reserve versus heart rate variability. The Journal of Trauma and Acute Care Surgery, 89(S2), S161-S168. doi:10.1097/TA.0000000000002605

Stengel, D., Leisterer, J., Ferrada, P., Ekkernkamp, A., Mutze, S., \& Hoenning, A. (2018). Point-of-care ultrasonography for diagnosing thoracoabdominal injuries in patients with blunt trauma. Cochrane Database of Systematic Reviews, 12(12), CD012669. doi:10.1002/14651858.CD012669.pub2

Tonglet, M. L., Minon, J. M., Seidel, L., Poplavsky, J. L., \& Vergnion, M. (2014). Prehospital identification of trauma patients with early acute coagulopathy and massive bleeding: Results of a prospective non-interventional clinical trial evaluating the Trauma Induced Coagulopathy Clinical Score (TICCS). Critical Care 18(6), 648. doi:10.1186/s13054-014-0648-0.

Van Sickle, C., Schafer, K., Mulligan, J., Grudic, G. Z., Moulton, S. L., \& Convertino, V. A. (2013). A sensitive shock index for real time patient assessment during simulated hemorrhage. Aviation, Space, and Environmental Medicine, 84(9), 907-912. doi: $10.3357 /$ asem.3606.2013

Vishwanathan, K., Chhajwani, S., Gupta, A., \& Vaishya, R. (2021). Evaluation and management of haemorrhagic shock in polytrauma: Clinical practice guidelines. Journal of Clinical Orthopaedics and Trauma, 13, 106-115. doi:10.1016/j. jcot.2020.12.003

Weeber, H., Hunter, L. D., Van Hoving, D. J., \& Lategan, H. (2018). Estimated injury-associated blood loss versus availability of emergency blood products at a district-level public hospital in Cape Town, South Africa. African Journal of Emergency Medicine, 8(2), 69-74. doi:10.1016/j.afjem.2018. 01.004

Williams, B., \& Boyle, M. (2007). Estimation of external blood loss by paramedics: Is there any point? Prehospital and Disaster Medicine, 22(6), 502-506. doi:10.1017//s1049023x0000532x.

World Health Organization. (2021). Injuries and violence: Fact sheet. World Health Organization. Retrieved June 22, 2021, from 
https://www.who.int/news-room/fact-sheets/detail/injuriesand-violence

Wyen, H., Lefering, R., Maegele, M., Brockamp, T., Wafaisade, A., Wutzler, S., ... Marzi, I. (2013). The golden hour of shock-how time is running out: Prehospital time intervals in Germany, a multivariate analysis of 15103 patients from the Trauma Register DGU. Emergency Medicine Journal, 30(12), 1048-1055. doi:10.1136/emermed-2012-201962
Zarzaur, B. L., Croce, M. A., Fischer, P. E., Magnotti, L. J., \& Fabian, P. C. (2008). New vitals after injury: Shock index for the young and age $\mathrm{x}$ shock index for the old. Journal of Surgical Research, 147(2), 229-236. doi:10.1016/j.jss.2008.03.025

Zia, J., Kimball, J., Rolfes, C., Hahn, J. O., \& Inan, O. T. (2020). Enabling the assessment of trauma-induced hemorrhage via smart wearable systems. Science Advances, 6(30), eabb1708. doi:10.1126/sciadv.abb1708 